\title{
Selective domain wall depinning by localized Oersted fields and Joule heating
}

\author{
Dennis Ilgaz, ${ }^{1}$ Mathias Kläui, ${ }^{1, a)}$ Lutz Heyne, ${ }^{1}$ Olivier Boulle, ${ }^{1}$ Fabian Zinser, ${ }^{1}$ \\ Stephen Krzyk, ${ }^{1}$ Mikhail Fonin, ${ }^{1}$ Ulrich Rüdiger, ${ }^{1}$ Dirk Backes, ${ }^{2, b)}$ and Laura J. Heyderman ${ }^{2}$ \\ ${ }^{1}$ Fachbereich Physik, Universität Konstanz, Universitätsstr. 10, D-78457 Konstanz, Germany \\ ${ }^{2}$ Laboratory for Micro- and Nanotechnology, Paul Scherrer Institut, CH-5232 Villigen PSI, Switzerland
}

(Received 27 June 2008; accepted 6 September 2008; published online 2 October 2008)

\begin{abstract}
Using low temperature magnetoresistance measurements, the possibility to selectively move a domain wall locally by applying current pulses through a Au nanowire adjacent to a permalloy element is studied. We find that the domain wall depinning field is drastically modified with increasing current density due to the Joule heating and the Oersted field of the current, and controlled motion due to the Oersted field without any externally applied fields is achieved. By placing the domain wall at various distances from the Au wire, we determine the range of the Joule heating and the Oersted field and both effects can be separated. (C) 2008 American Institute of Physics. [DOI: 10.1063/1.2990629]
\end{abstract}

The behavior of geometrically confined domain walls (DWs) and wall motion due to applied fields and current pulses has become a topic of growing interest in the past years. DW motion driven by applied magnetic fields ${ }^{1}$ or due to the spin transfer torque when current pulses are injected has been investigated. ${ }^{2-4}$ So far global external fields have been used to displace DWs, but for the control of a single DW, localized fields, or localized depinning is necessary, for which different schemes have been devised. ${ }^{5-7}$ The following investigation focuses on the DW motion influenced by local Oersted fields and Joule heating resulting from a current pulse in an adjacent wire. This topic attracts attention because of its potential in developing technological devices such as the race track memory ${ }^{6}$ or the magnetic shift register based on local thermal activation [for explanations and a schematic, see Figs. 1(a)-1(d)]. ${ }^{7}$ To implement such a scheme, localized ways to control the DW motion have to be devised. In this letter we report on the dependence of DW depinning fields on Joule heating and Oersted field due to currents in wires in the vicinity of a DW. We ascertain the contributions to the change in the DW displacement field from the Oersted field associated with the current and from the heating due to Ohmic losses in the adjacent wire. The understanding of this interplay then allows us to tailor the DW behavior.

The sample is fabricated by molecular beam epitaxy and a double lift-off process. ${ }^{8}$ The magnetic material permalloy (Py) with a capping layer of $\mathrm{Au}\left[\mathrm{Fe}_{20} \mathrm{Ni}_{80}(40 \mathrm{~nm}) /\right.$ $\mathrm{Au}(2 \mathrm{~nm})]$ and $20 \mathrm{~nm}$ thick Au contacts are deposited on a $\mathrm{Si}$ wafer. The sample has no artificial pinning centers due to notches but rather pinning centers due to roughness and other material defects. The structure of interest is a $500 \mathrm{~nm}$ wide permalloy half ring with five Au wires connected to it [Fig. 1(e)]. The central Au wire with a cross section of 300 $\times 20 \mathrm{~nm}^{2}$ is connected to a pulse generator, and the other four $\mathrm{Au}$ contacts are used for the magnetoresistance (MR) measurements [Fig. 1(f)]. Due to the dimensions of the per-

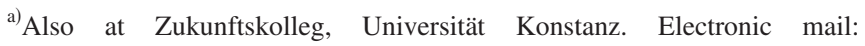
mathias.klaeui@uni-konstanz.de.

b) Also at Fachbereich Physik, Universität Konstanz, Universitätsstr. 10, D-78457 Konstanz, Germany.
}

malloy half ring, we expect vortex DWs to be present. ${ }^{4}$

The experimental setup is designed for magnetotransport measurements inside a cryostat at $4.3 \mathrm{~K}$. To position the DW between the voltage contacts (Au wires next to the central Au wire), we apply a field of $5000 \mathrm{G}$ in a specific direction, reduce this field to zero, and measure the resistance. ${ }^{9}$ Due to the anisotropic MR, the measured resistance depends on whether there is a DW between these Au wires (low resistance) or not (high resistance). ${ }^{9}$ In Fig. 2(a), four different resistance levels are visible for different wall positions. The highest levels correspond to the resistance when the DW is outside the region between the voltage contacts $\left(<73^{\circ}\right.$ and $>104^{\circ}$ ), for example positions 1 and 5 in Fig. 1(f). The resistance is lower when the DW is located at position 2 between the voltage contacts $\left(74^{\circ}-85^{\circ}\right)$. The central level 3 shows a small increase in the resistance in the range from $86^{\circ}-93^{\circ}$ when the DW is located at the $\mathrm{Au} / \mathrm{Py}$ interface below the central Au wire, which shunts a part of the current for the MR measurement (position 3). In the range from $94^{\circ}-102^{\circ}$, the DW is held at a pinning center partly below the $\mathrm{Au} / \mathrm{Py}$ interface (position 4) giving a resistance higher than that at position 2 . (a)

(b)

(c)

(d)

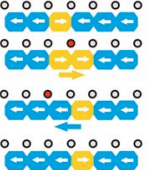

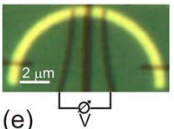

(e)
FIG. 1. (Color online) [(a)-(d)] Schematic of a shift register in which domains are moved by using thermally activated DW traps. (a) The colors yellow and blue correspond to different magnetization directions. (b) A wire with periodically arranged notches exhibits different domain configurations between the traps. By applying a field [arrow in (b)] and heating one specific notch (red dot) the DW can be selectively depinned at this notch. (c) By heating a different notch and applying a field in the opposite direction [arrow in (c)], the DW is depinned at that notch and is caught at the next notch. Thus the domain is displaced one step forward (d) using this slip-stick motion (Ref. 7). (e) Optical micrograph of the sample. The bright wire is Py and the dark wires are Au. (f) In this schematic, the relevant sample features are shown. The DW is placed in the magnetic Py half ring. The central Au wire is used for current pulses and the four outer $\mathrm{Au}$ wires are used for the MR measurement [central Au wire and voltage contacts are shown in (f)]. The different positions of the DWs are indicated by numbers 1-5. 


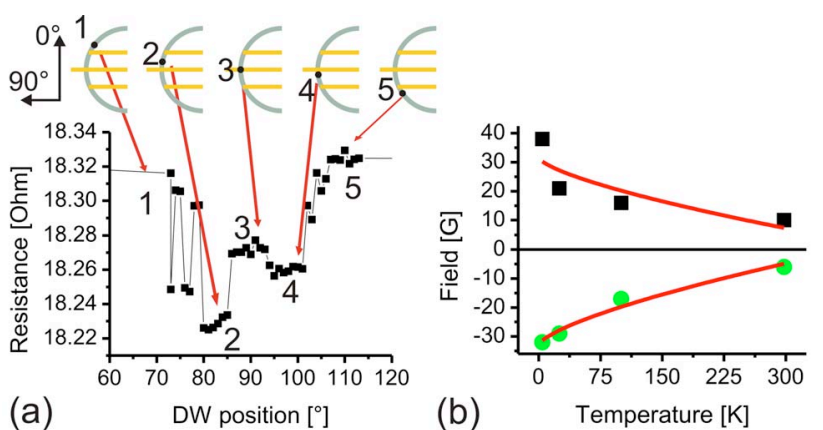

FIG. 2. (Color online) (a) MR measurement at $4.3 \mathrm{~K}$ as a function of the angle of the saturation field (as detailed in Ref. 9). The DW positions are labeled by numbers, which correspond to those in Fig. 1. The corresponding DW positions are indicated in the schematics above. In the field direction range between $73^{\circ}$ and $80^{\circ}$, we find that the wall can sit either at position 1 or 2 with a certain probability, which means that if the measurement is repeated for these angles, the resistance can be either high or low. For a single measurement as shown in (a), this results in the apparent noise between $73^{\circ}$ and $80^{\circ}$, and to obtain reliable results, this angle range is not used in the later measurement. (b) Temperature dependence of depinning fields $H_{d}$ for a positive field (black squares) and a negative field (green dots) for the DW placed at $98^{\circ}$ (position 4 ). The red line is a fit curve using the model of Kurkijärvi (Ref. 10).

In the next step, the field for the depinning of the DW from the pinning site at position 4 is studied. This depends on the angle of the applied field and is minimal if the field points perpendicularly to the central $\mathrm{Au}$ wire (at $0^{\circ}$ or $180^{\circ}$ ). Pinning centers due to edge roughness or other defects increase the depinning field of the DW. ${ }^{4}$ To move the DW, a depinning field $H_{d}$ needs to be applied, which depends on the temperature. $H_{d}(T)$ can be approximated by ${ }^{10,11}$

$$
\begin{aligned}
H_{d}(T) \cong & H_{0}\left\{1-\left[k_{b} T / E_{0} \ln \left(\Gamma_{0} k_{b} T H_{0} / 1.5 E_{0} v(1\right.\right.\right. \\
& \left.\left.\left.\left.-H_{d} / H_{0}\right)^{1 / 2}\right)\right]^{2 / 3}\right\} .
\end{aligned}
$$

The parameters are the field sweep rate $(v)$, the attempt frequency at zero temperature $\left(\Gamma_{0}\right)$, the depinning field at $0 \mathrm{~K}$ $\left(H_{0}\right)$, and the energy barrier $\left(E_{0}\right)$ for no applied field. This equation was derived by Lok et al. ${ }^{10}$ and stems from the Kurkijärvi model.

To depin the DW, we apply an external in-plane field, which is perpendicular to the central Au wire. In Fig. 2(b), the results for the depinning field at different cryostat temperatures are presented. The lowering of the depinning field due to thermal activation is visible. To obtain the energy barrier $E_{0}$ from Eq. (1), we use for the parameters $\Gamma_{0}$ $=10^{9} \mathrm{~Hz}, v=2 \mathrm{Oe} / \mathrm{s}$, and the relation of $H_{d} / H_{0}=0.58$. This ratio is the average value of $H_{d} / H_{0}$ at 4.3 and $298 \mathrm{~K}$, but the result for $E_{0}$ is not very sensitive to the ratio $H_{d} / H_{0}$. For a positive field, we find $E_{0}=1.24 \times 10^{-19} \mathrm{~J}$, and for a negative field, $E_{0}=1.07 \times 10^{-19} \mathrm{~J}$, which is in line with earlier experiments. ${ }^{11,12}$

Next we determine the change in the depinning field of a DW for different current pulses through the central Au wire with a current density of up to $6.6 \times 10^{11} \mathrm{~A} / \mathrm{m}^{2}$ at $4.3 \mathrm{~K}$. Here we do not inject any current into the Py. We focus on a DW position below the central Au wire placed by a negative field in the direction of $90^{\circ}$ [position 3, Fig. 2(a)] and on an adjacent position $150 \mathrm{~nm}$ away from the central wire placed by a positive field in a direction of $82^{\circ}$ [position 2, Fig. 2(a)]. The data points in Fig. 3 correspond to the first DW motion away from its original position and the DW motion out of the area between the voltage contacts.

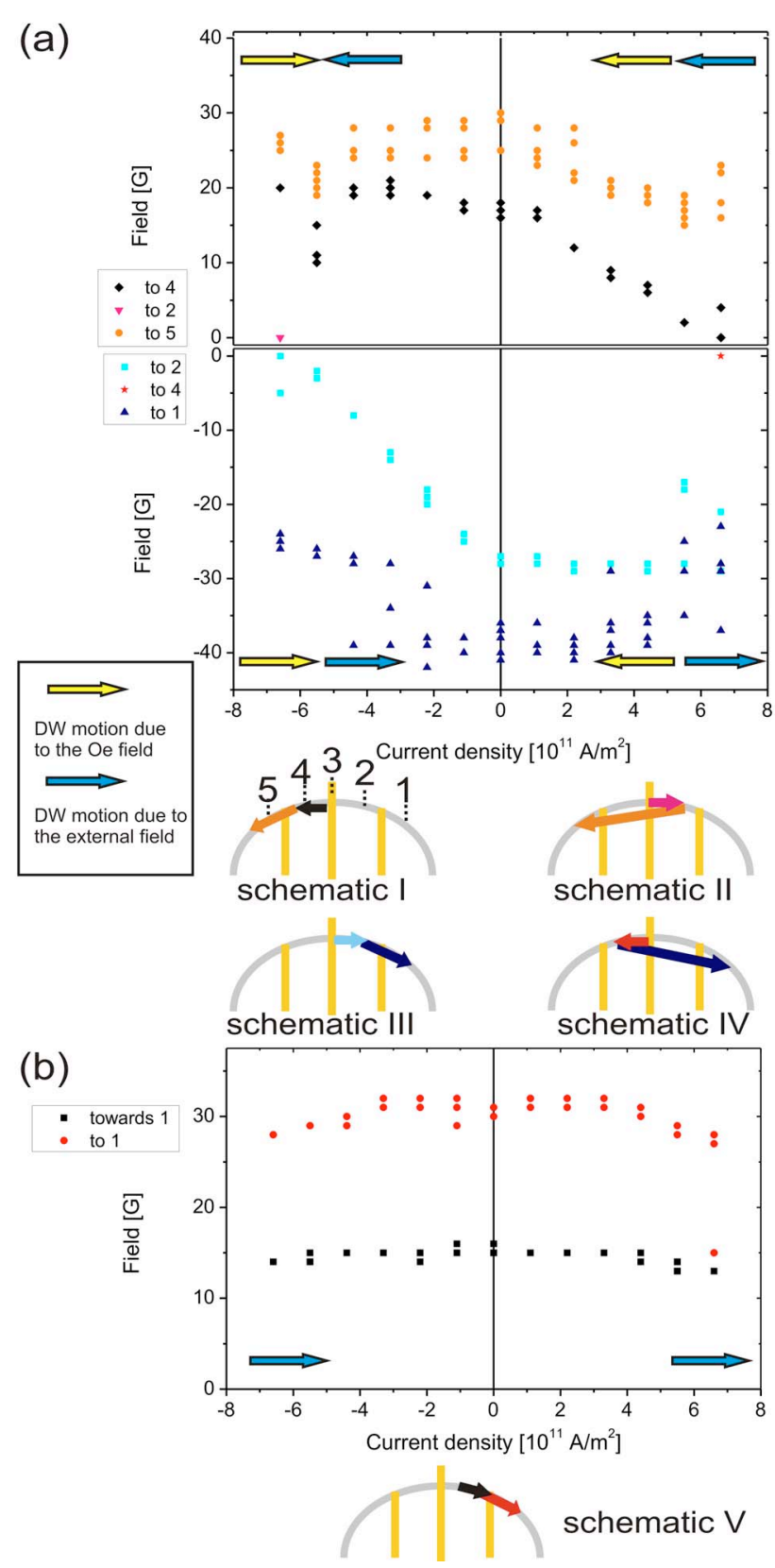

FIG. 3. (Color online) DW propagation fields for different applied fields and current pulses for DWs positioned originally at $90^{\circ}$ [position 3, (a)] and $82^{\circ}$ [position 2, (b)]. The DW motion direction due to the external field is indicated by the blue arrows, and the direction due to the Oersted field is indicated by the yellow arrows. (a) Positive field: the black diamonds indicate the first displacement from position 3 to position 4 . The orange disks indicate a DW motion from position 4 to 5 . The pink down triangle indicates a displacement from position 3 to position 2 . The data in (a) negative field show the corresponding results for a reversed applied field. Turquoise squares indicate the displacement from position 3 to 2 , blue up triangles indicate the displacement to position 1 , and the red star is the displacement from position 3 to 4 . (b) shows the result for a DW placed at $82^{\circ}$. The schematics below the graphs visualize the DW motion direction in the sample.

First the DW is positioned underneath the central Au wire along $90^{\circ}$ (position 3). Figure 3(a) shows the effect of an applied field along $0^{\circ}$, which favors motion of the DW toward position 5 and current pulses in two different directions, which causes an Oersted field of different signs. A positive current density corresponds to an Oersted field direction in the same direction as the external field. Without current, there are two main movements of the DW. The first 
displacement is from position 3 (Py/Au-interface) at about $18 \mathrm{G}$ to a pinning site at position 4 (black diamonds). The second displacement to the area outside the contacts occurs at about $25 \mathrm{G}$ (orange disks), and these movements are visualized in the schematic $I$ below the graph [Fig. 3(a)]. Upon applying positive current pulses, the depinning field for the first DW movement (from position 3 to 4) decreases with increasing current density since the Oersted field of the current pulse through the central Au wire supports the wall motion due to the external field. At a maximum current density of $6.6 \times 10^{11} \mathrm{~A} / \mathrm{m}^{2}$, the wall moves purely due to the Oersted field with no externally applied field. The fact that the second DW displacement field (orange disks) also decreases slightly means that the Oersted field extends to position 4, which is a sign of a pinning center next to the central $\mathrm{Au}$ wire. For negative current pulse densities up to -4.4 $\times 10^{11} \mathrm{~A} / \mathrm{m}^{2}$, the depinning field for the first motion from position 3 to 4 does not change much. The Oersted field hinders the field-induced DW motion and the DW moves only due to the applied field since the current is pulsed. For a very large negative current density $\left(-6.6 \times 10^{11} \mathrm{~A} / \mathrm{m}^{2}\right)$ the wall moves due to the Oersted field at zero applied external field (pink down triangle). In this case, the wall first moves to position 2 (pink down triangle), which is in the opposite direction to the external field, and then due to the external field to position 5 (orange disks) as shown in schematic II in Fig. 3.

Next we apply a field along $180^{\circ}$, which moves the DW toward position 1 [negative fields in Fig. 3(a)]. The DW moves from position 3 to position 2 (turquoise squares) and to the area outside the voltage contacts (blue up triangles, see Fig. 3 schematic III). The first motion from position 3 to position 2 is at about $-27 \mathrm{G}$ (turquoise squares). The motion to the area outside the voltage contacts occurs at about $-38 \mathrm{G}$ (blue up triangles). We find analogous behavior for the first DW movements from position 3 to 2 as for the field along $0^{\circ}$ in Fig. 3(a); negative current pulses create an Oersted field that lowers the depinning field for this external field direction. When applying negative current pulses, DW displacements from position 2 to position 1 [Fig. 3(a) blue up triangles] exhibit two different depinning fields. For low current densities, a displacement is observed at $-38 \mathrm{G}$ and higher current densities at $-27 \mathrm{G}$. This is because of slightly different DW positions and switching modes, which are not separated. We find that for the displacement at around $-27 \mathrm{G}$ for negative current densities up to -6.6 $\times 10^{11} \mathrm{~A} / \mathrm{m}^{2}$, there is no large decrease in the depinning field with increasing current density (and thus Oersted field) visible in contrast to Fig. 3(a) (orange disks). That means that in the first movement, the DW moved further away from the $\mathrm{Au}$ wire so that the Oersted field does not reach far enough to yield a decrease in the second displacement field. For positive current pulses, there is no significant decrease in the depinning field for the first motion from position 3 to 2 . The DW motion occurs if the applied field is high enough, just as in the case without a current pulse. For very high current densities, the Oersted field moves the DW to position 4 (red star) (see schematic IV in Fig. 3). These results show that the Oersted field created by the current pulse in the $\mathrm{Au}$ wire has a larger effect on DWs directly underneath the $\mathrm{Au}$ wire than Joule heating, but only for a relatively short range. The short range of the Oersted field is also indicated by the fact that the wall does not move directly to position 1 .

Now we place the DW about $150 \mathrm{~nm}$ away from the central wire at $82^{\circ}$. We consider applied fields [Fig. 3(b)] that move the DW away from the central wire to a pinning center close to position 1 (see Fig. 3 schematic V). Here we find a depinning field that decreases symmetrically with positive and negative current directions. This indicates a pure Joule heating effect and no effect of the Oersted field, which is asymmetric with respect to the current polarity (see before). The depinning field is $H_{0}=15 \mathrm{G}$ and $H_{d}=13 \mathrm{G}$ for the first motion (black squares) and $H_{0}=30 \mathrm{G}$ and $H_{d}=27 \mathrm{G}$ for the second one (red disks). With these parameters, we use Eq. (1) to obtain the temperature rise from $4.3 \mathrm{~K}$ (cryostat temperature) to $24 \mathrm{~K}$ for the black squares and to $15 \mathrm{~K}$ for the red disks. To investigate the heating in more detail, we determine the temperature of the central $\mathrm{Au}$ wire during current injection and find an increase in temperature of about 110 $\mathrm{K} .{ }^{13}$ This means that the temperature drops by about $90 \mathrm{~K}$ within $150 \mathrm{~nm}$ so that Joule heating can also be treated as a rather localized effect.

Finally we have also investigated the same effects for opposite wall placement fields that generate tail-to-tail rather than head-to-head walls and we observe no difference, as expected.

With the finding that the Oersted field also has a rather short range, we can conclude that by using Au wires, we can locally tailor the wall motion behavior by injecting appropriate current pulse heights and polarities. Due to the short range of the Joule heating and Oersted field effects, DWs positioned more than $150 \mathrm{~nm}$ away are not significantly affected so that short distances between walls and thus high storage densities can be achieved. Furthermore by appropriately designing the pinning sites at short enough distances, ${ }^{12}$ we can move walls from one pinning site to the next by just using the Oersted field with no externally applied field. This opens up an avenue for simple device operation, e.g., pinning with notches in a ferromagnetic wire and conducting nonmagnetic wires at the position of the notches that generate controlled local fields to implement a full device.

The authors acknowledge support by the DFG (SFB 513 and 767) and the Samsung Advanced Institute of Technology.

\footnotetext{
${ }^{1}$ M. Hara, T. Kimura, and Y. Otani, Appl. Phys. Lett. 90, 242504 (2007). ${ }^{2}$ A. Yamaguchi, T. Ono, S. Nasu, K. Miyake, K. Mibu, and T. Shinjo, Phys. Rev. Lett. 92, 077205 (2004).

${ }^{3}$ M. Kläui, C. A. F. Vaz, J. A. C. Bland, W. Wernsdorfer, G. Faini, E. Cambril, L. J. Heyderman, F. Nolting, and U. Rüdiger, Phys. Rev. Lett. 94, 106601 (2005).

${ }^{4}$ M. Kläui, J. Phys.: Condens. Matter 20, 313001 (2008).

${ }^{5}$ C.-Y. You, Appl. Phys. Lett. 92, 152507 (2008); 92, 192514 (2008).

${ }^{6}$ S. S. P. Parkin, M. Hayashi, and L. Thomas, Science 320, 190 (2008).

${ }^{7}$ R. Cowburn, Imperial College London (patent pending).

${ }^{8}$ L. J. Heyderman, M. Kläui, B. Nöhammer, C. A. F. Vaz, J. A. C. Bland, and C. David, Microelectron. Eng. 73, 780 (2004).

${ }^{9}$ M. Kläui, C. A. F. Vaz, J. Rothman, J. A. C. Bland, W. Wernsdorfer, G. Faini, and E. Cambril, Phys. Rev. Lett. 90, 097202 (2003).

${ }^{10}$ J. G. S. Lok, A. K. Geim, U. Wyder, J. C. Manan, and S. V. Dubonos, J. Magn. Magn. Mater. 204, 159 (1999).

${ }^{11}$ A. Himeno, T. Okuno, T. Ono, K. Mibu, S. Nasu, and T. Shinjo, J. Magn. Magn. Mater. 286, 167 (2005).

${ }^{12}$ P. Dagras, M. Kläui, M. Laufenberg, D. Bedau, L. Vila, G. Faini, C. A. F. Vaz, J. A. C. Bland, and U. Rüdiger, J. Phys. D 40, 1247 (2007).

${ }^{13}$ M. Laufenberg, W. Bührer, D. Bedau, P.-E. Melchy, M. Kläui, L. Vila, G Faini, C. A. F. Vaz, J. A. C. Bland, and U. Rüdiger, Phys. Rev. Lett. 97, 046602 (2006).
} 